\title{
GRAPHENE
}

\section{Buckle or break}

The isolation of free-standing graphene sheets seems to contradict common belief about the existence of two-dimensional crystals. Monte Carlo simulations confirm that the sheets may be stabilized by the formation of finite-sized ripples.

Johan M. Carlsson

is in the Fritz Haber Institute of the Max Planck Society

Faradayweg 4-6, D-14195 Berlin, Germany

e-mail: johanc@fhi-berlin.mpg.de

Can two-dimensional crystals exist in our three-dimensional world? This question has been the topic of a number of theoretical studies in the past. Mermin and Wagner concluded that, because the periodic order of the atoms cannot be maintained in an infinite two-dimensional crystals, such crystals may not exist[1,2]. Similarly, elasticity theory has predicted that two-dimensional membranes are unstable at finite temperatures(>0K), such that large membranes suffer severe buckling $[3,4]$.

In contrast to these predictions are recent observations of individual sheets derived from layered materials such as $\mathrm{BN}, \mathrm{MoS}_{2}$ and graphite[5]. The exfoliation of single graphene layers from graphite[6], has given new life to our initial question. Graphene is a prime example if a two-dimensional crystal, as it is a single layer of carbon atoms and the building block of graphite and carbon nanotubes. The carbon atoms in a graphene sheet are depicted ideally as a flat hexagonal lattice, exactly one atomic layer thick contrary to the predictions by Mermin and Wagner. Whether a graphene sheet is completely flat or not, however, has been difficult to prove experimentally because it may crumple, like a piece of paper, if not treated carefully. Experimental reports that a free-hanging graphene sheet is buckled rather than flat[7] are therefore debated.

On page 858 of this issue, however, Fasolino et al. provide strong theoretical support for the experimental observations of ripples in graphene[8]. The researchers have performed large-scale Monte Carlo simulations in order to determine the equilibrium structure of an infinite single graphene sheet over a range of temperatures. The sheet spontaneously forms ripples with a characteristic wavelength as shown in Fig. 1 in Ref. 8, indicating that the ripples observed experimentally may not be an artifact but an intrinsic property.

To understand the origin of the ripples, the environment of the carbon atoms in graphene must be considered. Unlike a three-dimensional crystal, in graphene the carbon atoms do not have neighbouring atoms above or below the sheet, so there are no restoring forces from atoms in adjacent layers. The restoring forces perpendicular to the sheet are limited to the out-of-plane component of the forces between neighbouring atoms due to the bending of the sheet. These forces are much smaller than those along the bonds, such that the perpendicular vibrational modes are much softer than the in-plane modes. Thermal 
motion can then easily move the carbon atoms out of the plane, which could destroy the order in the graphene sheet even at very low temperature. To estimate the impact of these thermal fluctuations, the graphene sheet can be compared to a continuous membrane. Elasticity theory shows that there is a strain energy connected with the perpendicular displacements, since there is a coupling between bending and stretching of the membrane[9]. This strain energy limits the perpendicular displacements and keeps a finite membrane together. However, the membrane may become buckled at finite temperature as this is the most favorable way of diminishing the amplitude of the soft perpendicular vibrations. The connection between the vibration frequencies and the buckling can be understood by comparing a flat membrane to a nanotube. The acoustic vibration mode perpendicular to the membrane corresponds to the vibration of a nanotube in the radial direction. In smaller nanotubes with larger curvature, the tube is stiffer perpendicular to the wall and the frequency of the vibrations is higher. The membrane, in a similar way, becomes stiffer due to the buckling, which increases the frequency of the perpendicular vibrational modes.

The amplitude of the buckling, however, increases with the size of the membrane, because the restoring strain energy for a given deflection perpendicular to the membrane decreases with system size. Therefore, only small membranes are predicted to stay approximately flat, whereas macroscopic membranes would have significant ripples and infinite membranes would break up into smaller, finite membranes as a result of the thermal vibrations $[3,4]$.

The results of Fasolino and colleagues, however, show that a graphene sheet is neither a flat two-dimensional crystal nor a homogeneous and featureless membrane. Graphene instead forms spontaneous ripples with a characteristic wavelength of around $80 \AA$ at finite temperatures (Fig. 1 in Ref. 8). These ripples stand in stark contrast to the common belief that graphene is perfectly flat. The wavelength of the ripples corresponds to about 50 carbon-carbon bond lengths and this value falls well within the limits of the experimental estimate[7]. However, the calculated amplitude of the ripples is less than 1 $\AA$ even for an infinite sheet, in contrast to the prediction from elasticity theory. The reason is that graphene has an atomic structure that can respond to the thermal fluctuations by bond-length alternations in the plane. The calculated bond lengths deviate from the ideal value $1.42 \AA$ that is expected for bonds between $s p^{2}$-hybridized carbon atoms, and the deviation increases with temperature. The variation should be visible at room temperature where the bond-length distribution varies between $1.3 \AA$ similar to the average length of a double bond, up to $1.54 \AA$, which is the typical length of a single bond in diamond. This bond-length variation cannot be accommodated in the layer, so a slight displacement of the carbon atoms out of the plane occurs, leading to the small buckling observed. In addition, the variation in bond length leads to an overall lattice contraction that contributes to the bending rigidity. This limits the amplitude of the outof-plane ripples such that they are not threatening to destroy the graphene sheet.

The ripples may have an impact on the electronic properties of graphene. For example, it has been proposed that the ripples can be scattering centres[10] and limit conductivity in the plane. The wider implication of the study by Fasolino et al. is that two-dimensional 
crystals can exist in a three-dimensional world as long as they find a mechanism to limit the thermal motion of the atoms perpendicular to the crystal plane. For graphene, a buckling mechanism which originates from the flexibility of the carbon-carbon bonds provides the answer. Whether this mechanism could work for other lattices remains an open question, but these results suggest that the stability of two-dimensional crystals is not a closed topic.

References:

[1] Mermin N. D. and H. Wagner, Phys. Rev. Lett. 17, 1133 (1966).

[2] Mermin N. D., Phys. Rev. 176, 250 (1968).

[3] Nelson D. R. and Peliti L., J. Phys. 48, 1085 (1987).

[4] Doussal P. and Radzihovsky L., Phys. Rev. Lett. 69, 1209 (1992).

[5] Novoselov K., Jiang D.S., Schedin F., Booth T. J., Khotkevich V. V., Morozov S. V., and Geim A. K., Proc. Nat. Acad. Sci. 102, 10451 (2005).

[6] Novoselov K. S., Geim A. K., Morozov S. V., Jiang D., Zhang Y., Dubonos S. V., Grigorieva I. V., and Firsov A. A., Science 306, 666 (2004).

[7] Meyer J. C., Geim A. K., Katsnelson M. I., Novoselov K. S., Booth T. J., and Roth S., Nature 446, 60 (2007).

[8] Fasolino A, Los J. H., and Katsnelson M. I., Nature Materials 6, 858 (2007).

[9] Landau L. D. and Lifshitz E. M., Theory of Elasticity 3rd Ed. (1986), ButterworthHeinemann, Oxford, UK.

[10] Schedin F., Geim A. K., Morozov S. V., Blake P., Katsnelson M. I., and Novoselov K. S., Nature Materials 6, 652 (2007).

Published in Nature Materials 6, page 801 (2007).

(C) Nature Publishing Group, a division of the Macmillan Publishers Limited. 